Pacific

Journal of

Mathematics

FIBRATIONS ON BANACH MANIFOLDS

Olivia Gutú and Jesús A. Jaramillo

Volume $215 \quad$ No. 2

June 2004 


\title{
FIBRATIONS ON BANACH MANIFOLDS
}

\author{
Olivia Gutú and Jesús A. Jaramillo
}

\begin{abstract}
Let $f$ be a split submersion between paracompact Banach manifolds. We obtain here various conditions for $f$ to be a fiber bundle. First, we give general conditions in terms of path-liftings. As a consequence, we deduce several criteria: For example, $f$ is a fiber bundle provided it satisfies either some topological requirements (such as being a proper or a closed map) or, in the case of Finsler manifolds, some metric requirements (such as Hadamard integral condition).
\end{abstract}

\section{Introduction}

A classical theorem of Ehresmann [5] asserts that, if $M$ and $N$ are finitedimensional manifolds with $M$ paracompact and $N$ connected, every proper submersion $f: M \rightarrow N$ is a fiber bundle. This result was extended by Earle and Eells [4] to the case where $M$ and $N$ are Finsler manifolds modelled on Banach spaces, $M$ complete, and $f: M \rightarrow N$ is a proper surjective submersion with split kernels. In fact, Earle and Eells obtained a more general result (see [4, Theorem 3C]) in which the fiber bundle structure depends on the existence of a certain kind of right inverse of the differential $d f$. More recently, Rabier [14] extends the theorem of Ehresmann by proving that, if $M$ and $N$ are Finsler manifolds modelled on Banach spaces with $M$ complete and $N$ connected, and $f: M \rightarrow N$ is a "strong submersion with uniformly split kernels", then $f$ is a fiber bundle (see Section 4 for this terminology). A large number of applications and ramifications of this result are also discussed in [14]. On the other hand, Plastock [13] obtained conditions for a function $f$ to be globally equivalent to a projection, in the particular case that $f$ is a nonlinear Fredholm map between Banach spaces. Plastock used a powerful continuation method (the "method of line lifting"), which had proved to be also quite useful in problems of global inversion of functions (see e.g., [12], [15] and [7]).

In this paper we are concerned with the problem of giving conditions for a submersion to be a fibre bundle. Our purpose is to make a direct connection between the fiber bundle structure and suitable path-lifting properties. In this way, we obtain some fairly general results, formulated in terms of pathliftings, and from which all the above mentioned theorems can be derived as corollaries. The contents of the paper are as follows: In Section 2 we consider 
a split submersion $f: M \rightarrow N$ between paracompact Banach manifolds, $N$ connected. Note that no Finsler structure is needed here. We define the continuation property for $f$, and we prove that it implies that $f$ is a fiber bundle. Then, some consequences and variants of this result are given. In particular, we obtain that $f$ is a fiber bundle provided it is either a proper or a closed map (conditions which are in turn equivalent in this setting). In Section 3 we assume, in addition, that $M$ and $N$ are Finsler manifolds, $M$ complete. We introduce the bounded path-lifting property for $f$, proving that it is a sufficient condition for $f$ to be a fiber bundle. Then we describe several instances where $f$ has the bounded path-lifting property. For example, this is the case (for connected manifolds) when $f$ satisfies a Hadamard integral condition. This integral condition was first used by Hadamard [8] in problems of global inversion of functions, and it has been widely used in this context (see e.g., [12], [13], [11], [7] and references therein). On the other hand, the case where $f$ is a local diffeomorphism is also considered, and conditions are given here for $f$ to be a covering projection or a global diffeomorphism. Finally, Section 4 is devoted to submersions with uniformly split kernels in the sense of Rabier. We introduce a path-lifting condition adapted to this case, which allows us to apply our previous results.

\section{Fiber bundles via continuation property}

We start with some notations and definitions. Throughout this paper, $M$ and $N$ will denote $C^{2}$ paracompact Banach manifolds without boundary, modelled on Banach spaces $E$ and $F$, respectively. Following the terminology of [10], by $C^{k-}$ we mean " $C^{k-1}$ with $(k-1)$-th derivative locally Lipschitz". In this way, a cross section $\mathfrak{s}: M \rightarrow T M$ is said to be of class $C^{1-}$ if for each $x \in M$ there is a chart $\phi: V \rightarrow E$ at $x$, such that the map $d \phi\left(\phi^{-1}(\cdot)\right) \circ \mathfrak{s}\left(\phi^{-1}(\cdot)\right): \phi(V) \rightarrow E$ is locally Lipschitz.

Now let $f: M \rightarrow N$ be a $C^{1}$ map. Also according to [10], we say that $f$ is of class $C^{2-}$ if for each $x \in M$ there exist a chart $\phi: V \rightarrow E$ for $M$ at $x$ and a chart $\psi: W \rightarrow F$ for $N$ at $f(x)$, such that $f(V) \subset W$ and, if $\widetilde{f}:=\psi \circ f \circ \phi^{-1}: \phi(V) \rightarrow \psi(W)$, then the derivative $d \widetilde{f}: \phi(V) \rightarrow \mathcal{L}(E ; F)$ is locally Lipschitz. As it might be suspected, every map of class $C^{2}$ is of class $C^{2-}$ and the composition of $C^{2-}$ maps is of class $C^{2-}$. As usual, $f$ is said to be a split submersion if, for each $x \in M, d f(x) \in \mathcal{L}\left(T_{x} M ; T_{f(x)} N\right)$ is surjective and its kernel splits. It will be quite useful for us the possibility of "gluing together" continuous linear sections of each $d f(x)$ in a locally Lipschitz way. More precisely, we will say that $s(\cdot)$ is a $C^{1-}$ right inverse of $d f(\cdot)$ if the following two conditions are satisfied:

(i) For every $x \in M, s(x) \in \mathcal{L}\left(T_{f(x)} N ; T_{x} M\right)$ and $d f(x) \circ s(x)=\mathrm{id}$. 
(ii) For every chart $\phi: V \rightarrow E$ in $M$ and every chart $\psi: W \rightarrow F$ in $N$ with $f(V) \subset W$, the map

$$
d \phi\left(\phi^{-1}(\cdot)\right) \circ s\left(\phi^{-1}(\cdot)\right) \circ\left[d \psi\left(f\left(\phi^{-1}(\cdot)\right)\right)\right]^{-1}
$$

is locally Lipschitz in $\phi(V)$.

The next well-known lemma provides us the existence of such a kind of right inverse for $d f(\cdot)$. It depends on the fact that every paracompact Banach manifold admits partitions of unity of class $C^{1-}$ (see [10]). A direct proof for the case of a Fredholm submersion between Banach spaces is given in [13, Lemma 2.6].

Lemma 2.1 ([4, Lemma 3(B)]). Let $M$ and $N$ be $C^{2}$ paracompact Banach manifolds and let $f: M \rightarrow N$ be a split submersion of class $C^{2-}$. Then, there exists a $C^{1-}$ right inverse of $d f(\cdot)$.

Remark 2.2. In the special case that $M$ and $N$ are Hilbert manifolds, there is a canonical $C^{1-}$ right inverse of $d f(\cdot)$, which is given by an explicit formula. Namely

$$
s_{0}(x)=\left(\left.d f(x)\right|_{[\operatorname{ker} d f(x)]^{\perp}}\right)^{-1}=d f(x)^{*}\left[d f(x) d f(x)^{*}\right]^{-1},
$$

where $d f(x)^{*} \in \mathcal{L}\left(T_{f(x)} N ; T_{x} M\right)$ denotes the Hilbert space adjoint of $d f(x)$ (see [13, Lemma 2.5]).

Next we give the central result of this section. First, we need the following definition: We shall say that $f: M \rightarrow N$ has the continuation property if, for every $C^{1}$ path $p:[0,1] \rightarrow N$ and every $C^{1}$ path $q:[0, b) \rightarrow M$ with $f \circ q=p$, where $0<b \leq 1$, there exists an increasing sequence $t_{n} \rightarrow b$ such that the sequence $\left\{q\left(t_{n}\right)\right\}$ converges in $M$.

Theorem 2.3. Let $M$ and $N$ be $C^{2}$ paracompact Banach manifolds, $N$ connected. Let $f: M \rightarrow N$ be a split submersion of class $C^{2-}$. If $f$ has the continuation property then $f$ is a fiber bundle.

Proof. We are much inspired by the proof of Theorem 4.1 in [14]. First of all, consider a $C^{1-}$ right inverse $s(\cdot)$ of $d f(\cdot)$, which always exists by Lemma 2.1. Let $\left\{\left(W_{\kappa}, \psi_{\kappa}\right): \kappa \in \Lambda\right\}$ be an atlas of $N$. Without loss of generality, we can suppose that for every $\kappa \in \Lambda, \psi_{\kappa}\left(W_{\kappa}\right)=\widetilde{W}_{\kappa}$ is a ball centered at the origin in the Banach space $F$. For each $\kappa \in \Lambda$, denote $V_{\kappa}:=f^{-1}\left(W_{\kappa}\right) \subset M$.

Now, let us fix $\kappa \in \Lambda$ such that $V_{\kappa} \neq \emptyset$. Note that $V_{\kappa}$ is an open submanifold of $M$ and, for each $y \in F$, the cross section $\mathfrak{s}_{y}(\cdot):=s(\cdot) \circ\left[d \psi_{\kappa}(f(\cdot))\right]^{-1} y$ : $V_{\kappa} \rightarrow T V_{\kappa}$ is of class $C^{1-}$. For each $x \in V_{\kappa}$ and $y \in F$, if we consider the initial value problem:

$$
\begin{aligned}
\dot{q}(t) & =\mathfrak{s}_{y}(q(t)) \\
q(0) & =x
\end{aligned}
$$


we obtain that Equation (2.1) has a unique solution $q(t, x, y)$ in $V_{\kappa}$ over an open maximal interval $I_{x, y}=(a(x, y), b(x, y))$, with $0 \in I_{x, y}$. Furthermore the set

$$
\Omega=\bigcup_{(x, y) \in V_{\kappa} \times F} I_{x, y} \times\{(x, y)\}
$$

is open in $\mathbb{R} \times V_{\kappa} \times F$ and $q: \Omega \rightarrow V_{\kappa}$ is continuous (see $[\mathbf{1 0}, \S 1]$ ). Now let $(x, y) \in V_{\kappa} \times F$ be fixed. From Equation (2.1), it follows that $\dot{q}(t)=s(q(t)) \circ$ $\left[d \psi_{\kappa}(f(q(t)))\right]^{-1} y$ for every $t \in I_{x, y}$. If we denote $\widetilde{f}:=\left.\psi_{\kappa} \circ f\right|_{V_{\kappa}}: V_{\kappa} \rightarrow \widetilde{W}_{\kappa}$, we obtain that $\widetilde{f}(q(0))=\widetilde{f}(x)$ and $d \widetilde{f}(q(t))(\dot{q}(t))=y$ for every $t \in I_{x, y}$. As a consequence,

$$
\widetilde{f}(q(t, x, y))=\widetilde{f}(x)+t y, \quad \forall t \in I_{x, y} .
$$

Before going further, we will prove the following:

Claim. The continuation property implies that:

(i) If $x \in V_{\kappa}$, then $[-1,0] \in I_{x, \widetilde{f}(x)}$ and

(ii) if $(x, y) \in \widetilde{f}^{-1}(0) \times \widetilde{W}_{\kappa}$, then $[0,1] \in I_{x, y}$.

Indeed, for (i) let $x \in V_{\kappa}$, let $a:=a(x, \widetilde{f}(x))$ and suppose that $a \in[-1,0)$. Consider the line that joins 0 with $\widetilde{f}(x)$ in $\widetilde{W}_{\kappa}$, that is, $l(t)=(1-t) \widetilde{f}(x)$ for $t \in[0,1]$, and define the path $p=\psi_{\kappa}^{-1} \circ l:[0,1] \rightarrow N$. There is a path $\bar{q}(t):=q(-t, x, \widetilde{f}(x))$ on $[0,-a)$ such that $\widetilde{f}(\bar{q}(t))=l(t)$, for all $t \in[0,-a)$. Therefore $f \circ \bar{q}=p$ on $[0,-a)$, and by the continuation property, there is an increasing sequence $t_{n} \rightarrow-a$ such that $\bar{x}=\lim _{n} \bar{q}\left(t_{n}\right)$ exists in $M$. In fact, $\bar{x} \in V_{\kappa}$, since by continuity $\widetilde{f}(\bar{x})=l(-a) \in \widetilde{W}_{\kappa}$. Then, by the unique solvability of (2.1), $q$ can be extended outside $I_{x, \widetilde{f}(x)}$, contradicting its maximality. In consequence, $a<-1$ and in particular $q(-1, x, \widetilde{f}(x)) \in$ $\widetilde{f}^{-1}(0)$ and $\widetilde{f}^{-1}(0) \neq \emptyset$. For (ii) let $x \in \widetilde{f}^{-1}(0)$ and $y \in \widetilde{W}_{\kappa}$. Consider the line in $\widetilde{W}_{\kappa}$ given by $l(t)=\widetilde{f}(x)+t y=t y$, for $t \in[0,1]$. If $b(x, y) \leq 1$, by the same argument as before $q$ can be extended outside $I_{x, y}$, which is not possible. Therefore $q(1, x, y)$ is defined for every $x \in \widetilde{f}^{-1}(0)$ and $y \in \widetilde{W}_{\kappa}$.

Therefore we can define $\theta: \widetilde{f}^{-1}(0) \times \widetilde{W}_{\kappa} \rightarrow V_{\kappa}$ by $\theta(x, y)=q(1, x, y)$. We know that $\theta$ is continuous, and we are going to prove that it is a homeomorphism. Let $\left(x_{1}, y_{1}\right) \neq\left(x_{2}, y_{2}\right)$ in $\widetilde{f}^{-1}(0) \times \widetilde{W}_{\kappa}$ such that $q\left(1, x_{1}, y_{1}\right)=$ $q\left(1, x_{2}, y_{2}\right)$. By (2.2), $\widetilde{f}\left(x_{1}\right)+y_{1}=\widetilde{f}\left(x_{2}\right)+y_{2}$ and therefore $y_{1}=y_{2}=y$. In other words, $q\left(1, x_{1}, y\right)$ and $q\left(1, x_{2}, y\right)$ are values at time 1 of the differential equation (2.1) with initial values $x_{1}$ and $x_{2}$ respectively, and then $x_{1}=x_{2}$. In conclusion, $\theta$ is injective. On the other hand, let $x \in V_{\kappa}$. By the above arguments, we can choose $x^{\prime}=q(-1, x, \widetilde{f}(x)) \in \widetilde{f}^{-1}(0)$ and we obtain that $\theta\left(x^{\prime}, \widetilde{f}(x)\right)=x$. Then $\theta$ is surjective. Furthermore, the map 
$x \mapsto(q(-1, x, \widetilde{f}(x)), \widetilde{f}(x))$ is the continuous inverse of $\theta$. Note that, for every $y \in \widetilde{W}_{\kappa}$, the map $\theta(\cdot, y)$ is a homeomorphism from $\widetilde{f}^{-1}(0)$ onto $\widetilde{f}^{-1}(y)$. Thus for each $w \in W_{\kappa}$ we have that $f^{-1}(w)=\widetilde{f}^{-1}\left(\psi_{\kappa}(w)\right)$ is homeomorphic to $\mathfrak{F}_{\kappa}:=\widetilde{f}^{-1}(0)$. Now the map

$$
\Theta_{\kappa}:=\theta \circ\left(\mathrm{i} d \times \psi_{\kappa}\right): \mathfrak{F}_{\kappa} \times W_{\kappa} \rightarrow V_{\kappa}=f^{-1}\left(W_{\kappa}\right)
$$

is a homeomorphism and if $(x, w) \in\left(\mathfrak{F}_{\kappa}, W_{\kappa}\right)$, then $f\left(\Theta_{\kappa}(x, w)\right)=w$, that is, $f \circ \Theta_{\kappa}$ is the natural projection.

Next we will show that all the fibers of $f$ are homeomorphic. Note that this implies that $f$ is onto, and $V_{\kappa} \neq \emptyset$ for every $\kappa \in \Lambda$. In this way, we complete the proof that $f: M \rightarrow N$ is a fiber bundle. Let $\kappa_{0} \in \Lambda$ be fixed such that $V_{\kappa_{0}} \neq \emptyset$ and consider $N_{0}=\left\{w \in N: f^{-1}(w) \approx \mathfrak{F}_{\kappa_{0}}\right\}$, where $\approx$ denotes "is homeomorphic to". As we noticed before, $W_{\kappa_{0}} \subset N_{0}$. Given $w \in$ $N_{0}$, there exists $\kappa \in \Lambda$ such that $w \in W_{\kappa}$, and then $f^{-1}(v) \approx f^{-1}(w) \approx \mathfrak{F}_{\kappa_{0}}$ for all $v \in W_{\kappa}$. Therefore $N_{0}$ is open in $N$. In the same way, it can be seen that the complement of $N_{0}$ is open in $N$. By connectedness, we get that $N_{0}=N$.

A particular case of split submersion is obtained when $f: M \rightarrow N$ is a local diffeomorphism. In this case, if $f$ is a fiber bundle then the fibre is discrete, and therefore $f$ is a covering projection. On the other hand, every covering projection has the unique path lifting property, and as a consequence it also has the continuation property. Thus by Theorem 2.3 we have the following:

Corollary 2.4. Let $M$ and $N$ be $C^{2}$ paracompact Banach manifolds, $N$ connected. Let $f: M \rightarrow N$ be a $C^{2-}$ map such that

$$
d f(x) \in \operatorname{Isom}\left(T_{x} M ; T_{f(x)} N\right),
$$

for every $x \in M$. Then $f$ has the continuation property if and only if it is a covering projection.

If $f: M \rightarrow N$ is a fiber bundle and $N$ is contractible, it is well-known that $f$ is a trivial fiber bundle (see, e.g., [1, Theorem 3.4.35 ]). So, we have the following immediate consequence of Theorem 2.3, which can be seen as a global implicit function theorem:

Corollary 2.5. Let $M$ and $N$ be $C^{2}$ paracompact Banach manifolds, $N$ contractible. Let $f: M \rightarrow N$ be a split submersion of class $C^{2-}$. If $f$ has the continuation property, then $f$ is a trivial fiber bundle with trivialization $\mathfrak{F} \times N \approx M$.

Remark 2.6. Suppose that $f: E \rightarrow F$ is a split submersion of class $C^{2-}$ between Banach spaces, which satisfies the continuation property. In this situation, the proof of Theorem 2.3 can be considerably simplified, and it gives 
directly a global trivialization. Following the proof without using charts, we search for a right inverse $s(x) \in \mathcal{L}(F ; E)$ of $d f(x) \in \mathcal{L}(E ; F)$ and we consider Equation $(2.1)$ with $\mathfrak{s}_{y}(\cdot)=s(\cdot) y$, for $y$ fixed. The continuation property gives us the existence of the flow in the appropriate interval. In fact, it is enough here to lift lines, as in the method of line lifting used by Plastock in [13] for the case of a $C^{2-}$ Fredholm map. In conclusion, there exist a map $\Theta$ and a fiber $\mathfrak{F}$ such that: $\Theta: \mathfrak{F} \times F \rightarrow E$ is a homeomorphism and the composition $f \circ \Theta: \mathfrak{F} \times F \rightarrow F$ is the natural projection.

Recall that a continuous map $f: M \rightarrow N$ is said to be proper if, for every compact subset $K$ of $N$, the set $f^{-1}(K)$ is compact in $M$. More generally, we say that $f$ is weakly proper if, for every compact subset $K$ of $N$, each connected component of $f^{-1}(K)$ is compact in $M$. Now we can deduce, as a direct consequence of Theorem 2.3, the following extension of classical Ehresmann Theorem [5] to the infinite dimensional setting. In [14] Rabier gives an analogous result for proper maps in the case of Finsler manifolds.

Theorem 2.7. Let $M$ and $N$ be $C^{2}$ paracompact Banach manifolds, $N$ connected. If $f: M \rightarrow N$ is a weakly proper split submersion of class $C^{2-}$, then $f$ is a fiber bundle.

Proof. It is easy to check that if $f$ is weakly proper then it has the continuation property. Indeed, let $p:[0,1] \rightarrow N$ be a $C^{1}$ path, consider $0<b \leq 1$, and suppose that $q:[0, b) \rightarrow M$ satisfies $f \circ q=p$ over $[0, b)$. Then we have that $\operatorname{Im} q$ is relatively compact in $M$, since it is contained in a connected component of set $f^{-1}(\operatorname{Im} p)$. If we now choose an increasing sequence $t_{n} \rightarrow b$, there exists a subsequence $\left(t_{n_{k}}\right)$ such that $\left\{q\left(t_{n_{k}}\right)\right\}$ is convergent in $M$.

A slight variation in the proof of Theorem 2.3 gives the following result (compare with $[\mathbf{3}]$ ):

Theorem 2.8. Let $M$ and $N$ be $C^{2}$ paracompact Banach manifolds, $N$ connected. If $f: M \rightarrow N$ is a closed split submersion of class $C^{2-}$, then $f$ is a fiber bundle.

Proof. If we follow the proof of Theorem 2.3, we see that the continuation property is only used in the Claim. Therefore, in order to reach the same conclusion, it is enough to prove the following (where we maintain the notation of Theorem 2.3):

Claim. The closedness of $f$ implies that

(i) if $x \in V_{\kappa}$, then $[-1,0] \in I_{x, \widetilde{f}(x)}$ and

(ii) if $(x, y) \in \widetilde{f}^{-1}(0) \times \widetilde{W}_{\kappa}$, then $[0,1] \in I_{x, y}$.

Indeed, for (i) let $x \in V_{\kappa}$, let $a:=a(x, \widetilde{f}(x))$ and suppose that $a \in[-1,0)$. Consider the path $p=\psi_{\kappa}^{-1} \circ l:[0,1] \rightarrow N$, where $l(t)=(1-t) \widetilde{f}(x)$. There 
is a path $\bar{q}(t):=q(-t, x, \widetilde{f}(x))$ on $[0,-a)$ such that $f \circ \bar{q}=p$ on $[0,-a)$. Let $C$ be the closure of $\operatorname{Im} \bar{q}$ in $M$. Since $f(C)$ is closed in $N$ and it contains $p(t)$ for every $t \in[0,-a)$, there exists $\bar{x} \in C$ such that $f(\bar{x})=p(-a)$. Therefore, there is a sequence $\left(t_{n}\right)$ in $[0,-a)$ such that $\bar{q}\left(t_{n}\right) \rightarrow p(-a)$, and we can also assume that $\left(t_{n}\right)$ is convergent to some $\bar{t} \in[0,-a]$. By continuity, $p(\bar{t})=p(-a)$, and since $p$ is one-to-one we have that $\bar{t}=-a$. Then, by the unique solvability of (2.1), $q$ can be extended outside $I_{x, \widetilde{f}(x)}$, contradicting its maximality. As a consequence, $a<-1$. For (ii), the argument is similar.

From the above theorem it follows that properness and closedness are in fact equivalent in our setting. More precisely, we have:

Corollary 2.9. Let $M$ and $N$ be $C^{2}$ paracompact Banach manifolds, with $N$ connected, and let $f: M \rightarrow N$ be a split submersion of class $C^{2-}$. Then $f$ is proper if, and only if, $f$ is closed.

Proof. In general, every proper map $f: M \rightarrow N$ is closed. Conversely, suppose that $f$ is closed. By Theorem 2.8 we have that $f$ is a fiber bundle. In order to prove that $f$ is proper, it is sufficient to show that the fiber is compact (see, e.g., [6, Theorem 3.7.2]). Indeed, if this is not the case, the fiber contains a sequence with no convergent subsequence. Using this and considering a convergent sequence $\left(y_{n}\right)$ in $N$ with pairwise different terms, it is easy to construct a sequence $\left(x_{n}\right)$ in $M$ with no convergent subsequence and such that $f\left(x_{n}\right)=y_{n}$. This contradicts the closedness of $f$, since the set $C=\left\{x_{n}: n \in \mathbb{N}\right\}$ is closed but $f(C)$ is not.

On the other hand, we note that properness (or closedness) is in fact a quite restrictive condition in this situation. For example, Berger and Plastock [2] proved that, in the case of functions between Banach spaces, there is no $C^{2-}$ proper Fredholm submersion with index $\geq 1$. More generally we obtain that, in the case of a $C^{2-}$ split submersion between contractible manifolds, properness (or closedness) is in fact equivalent to global homeomorphism.

Corollary 2.10. Let $M$ and $N$ be $C^{2}$ paracompact, contractible Banach manifolds. If $f: M \rightarrow N$ is a split submersion of class $C^{2-}$, the following are equivalent:

(1) $f$ is a homeomorphism,

(2) $f$ is a closed map,

(3) $f$ is a proper map.

Proof. By Corollary 2.9, it only remains to prove that (3) implies (1). Suppose that $f$ is a proper map. By Theorem 2.7 we get that $f$ is a fiber bundle. Again by [1, Theorem 3.4.35 ], $f$ is actually a trivial fiber bundle with trivialization $M \approx \mathfrak{F} \times N$. Since $M$ is contractible, so is the fiber $\mathfrak{F}$. On the other hand, the properness of $f$ implies that the fiber $\mathfrak{F}$ is a compact submanifold 
of $M$ without boundary. Since there is no contractible compact $C^{1}$ manifold with positive dimension (see e.g., $[\mathbf{9}$, Theorem 5.1.6]), we obtain that $\mathfrak{F}$ is a singleton. Therefore, $f$ is a homeomorphism.

We conclude this section with a technical improvement of Theorem 2.3 which will be quite useful in the sequel. We need the following definition: Let $f: M \rightarrow N$ be a $C^{2-}$ split submersion and $s(\cdot)$ a fixed $C^{1-}$ right inverse of $d f(\cdot)$. Let $p:[0,1] \rightarrow N$ be a $C^{1}$ path, and $0<b \leq 1$. We shall say that a $C^{1}$ path $q:[0, b) \rightarrow M$ is an horizontal lifting of $p$ if $f \circ q=p$ on $[0, b)$ and:

$$
\dot{q}(t)=s(q(t)) \dot{p}(t), \quad \forall t \in[0, b) .
$$

Theorem 2.11. Let $M$ and $N$ be $C^{2}$ paracompact Banach manifolds, $N$ connected. Let $f: M \rightarrow N$ be a split submersion of class $C^{2-}$, and $s(\cdot)$ a fixed $C^{1-}$ right inverse of $d f(\cdot)$. If $f$ has the continuation property for horizontal liftings, then $f$ is a fiber bundle.

Proof. We note as before that, in the proof of Theorem 2.3, the continuation property is only used in the Claim. Therefore, in order to obtain the same conclusion, it is enough to have the continuation property to hold, not for arbitrary paths, but only for solutions $q(t, x, y)$ of the initial value problem (2.1) where $x$ and $y$ are conveniently chosen. More precisely, with $x \in V_{\kappa}$ and $y=\widetilde{f}(x)$ in Case (i) and with $x \in \widetilde{f}^{-1}(0)$ and $y \in \widetilde{W}_{\kappa}$ in Case (ii). It is easy to see that, in both cases, these solutions are horizontal lifts. Indeed, for the first case, let $p(t):=\psi_{\kappa}^{-1}((1-t) \widetilde{f}(x))$ defined on $[0,1]$, and let $\bar{q}(t)=q(-t, x, \widetilde{f}(x))$ defined on the maximal half-interval $[0,-a)$. As we saw in the proof of Theorem 2.3, $f \circ \bar{q}=p$ over $[0,-a)$ and then $\dot{p}(t)=-\left[d \psi_{\kappa}(f(q(-t)))\right]^{-1} \widetilde{f}(x)$, for $t \in[0,-a)$. Therefore, $\dot{\bar{q}}(t)=-\dot{q}(-t)=$ $s(q(-t)) \circ \dot{p}(t)=s(\bar{q}(t)) \dot{p}(t)$ over $[0,-a)$. Case (ii) is analogous.

\section{Bounded path-lifting property}

In this section we will work in the framework of Finsler manifolds. Recall from [10] that a Finsler manifold $M$ is a Banach manifold that admits for its tangent bundle a functional $\|\cdot\|: T M \rightarrow \mathbb{R}$ satisfying the following two conditions:

(i) For every $x \in M$, the map $v \mapsto\|(x, v)\|$ is an admissible norm for $T_{x} M$, which we denote by $\|\cdot\|_{x}$.

(ii) Given $x_{0} \in M$, a chart $\phi: V \rightarrow E$ at $x_{0}$ in $M$ and $k>1$, there exists an open neighborhood $U_{x_{0}}$ of $x_{0}$ in $V$ such that

$$
k^{-1}\left\|d \phi^{-1}\left(\phi\left(x_{0}\right)\right) v\right\|_{x_{0}} \leq\left\|d \phi^{-1}(\phi(x)) v\right\|_{x} \leq k\left\|d \phi^{-1}\left(\phi\left(x_{0}\right)\right) v\right\|_{x_{0}}
$$

for all $x \in U_{x_{0}}$ and $v \in E$.

In this case, the length of a piecewise $C^{1}$ path $p:[a, b] \rightarrow M$ is defined by $\ell_{F}(p):=\int_{a}^{b}\|\dot{p}(t)\| d t$. If the domain of $p$ is a half-open interval $[a, b)$, 
then $\ell_{F}(p):=\lim _{t \rightarrow b^{-}} \ell_{F}\left(\left.p\right|_{[0, t]}\right)$. On each connected component of $M$, the Finsler metric is defined by

$$
d_{F}(x, y)=\inf \left\{\ell_{F}(p): p \text { is a piecewise } C^{1} \text { path from } x \text { to } y\right\} .
$$

This metric is compatible with the topology of the corresponding component of $M$, see [10, Theorem 3.3]. Note in particular that, since each connected component of $M$ is open, $M$ is metrizable and therefore it is paracompact. Now $M$ is said to be complete if each connected component is complete with respect to its Finsler metric. For technical reasons, we are interested in defining a boundedness condition for path-liftings in terms of a weight. As we will see, this is useful in order to obtain a type of Hadamard integral condition. The relevance of such weights has been also noted in [14, Remark 4.4]. By a weight we mean an increasing monotonic map $\omega:[0, \infty) \rightarrow(0, \infty)$ such that:

$$
\int_{0}^{\infty} \frac{d t}{\omega(t)}=\infty
$$

Lemma 3.1. Let $M$ and $N$ be $C^{2}$ Finsler manifolds, $M$ complete and $N$ connected. Let $f: M \rightarrow N$ be a $C^{2-}$ split submersion and $s(\cdot)$ a fixed $C^{1-}$ right inverse of $d f(\cdot)$. Consider a $C^{1}$ path $p:[0,1] \rightarrow N$, a $C^{1}$ horizontal lifting $q:[0, b) \rightarrow M$ of $p$, where $0<b \leq 1$, and let $M_{0}$ be the connected component of $M$ containing $\operatorname{Im} q$. Then the following conditions are equivalent:

(1) There exists $C>0$ such that $\sup \{\|s(x)\|: x \in \operatorname{Im} q\} \leq C$.

(2) There exist $C>0$, some weight $\omega$, and some $x_{0} \in M_{0}$ such that

$$
\sup \left\{\frac{\|s(x)\|}{\omega\left(d_{F}\left(x, x_{0}\right)\right)}: x \in \operatorname{Im} q\right\} \leq C .
$$

(3) $\ell_{F}(q)<\infty$.

(4) The path $q$ can be extended continuously to $[0, b]$.

(5) $\overline{\operatorname{Im} q} \subset M$ is compact.

Proof. $(1 \Rightarrow 2)$ Is obvious, by taking $\omega(t) \equiv 1$.

$(2 \Rightarrow 3)$ We can suppose, with no loss of generality, that $x_{0}=q(0)$ (since otherwise we could consider the alternative weight $\bar{\omega}(t):=\omega\left(d_{F}\left(x_{0}, q(t)\right)+\right.$ $t)$ ). Let $0<\delta<b$ be fixed, and define the map $\xi:[0, \delta] \rightarrow \mathbb{R}$ by

$$
\xi(\tau)=\max _{t \in[0, \tau]} d_{F}\left(q(t), x_{0}\right) .
$$

It is clear that $\xi$ is continuous and non-decreasing. Before going further, we are going to show that:

$$
\xi(\tau)-\xi\left(\tau^{\prime}\right) \leq C \cdot \omega(\xi(\tau)) \cdot \ell_{F}\left(\left.p\right|_{\left[\tau^{\prime}, \tau\right]}\right), \quad \forall \tau^{\prime} \leq \tau .
$$


Indeed, let $\tau^{\prime}<\tau$ in $[0, \delta]$. From the equality (2.3) we get that for every $t \in\left[\tau^{\prime}, \tau\right]:$

$$
\|\dot{q}(t)\| \leq\|s(q(t))\| \cdot\|\dot{p}(t)\| .
$$

Therefore,

$$
\ell_{F}\left(\left.p\right|_{\left[\tau^{\prime}, \tau\right]}\right)=\int_{\tau^{\prime}}^{\tau}\|\dot{p}(t)\| d t \geq \inf \left\{\|s(x)\|^{-1}:\left.x \in \operatorname{Im} q\right|_{\left[\tau^{\prime}, \tau\right]}\right\} \cdot d_{F}\left(q\left(\tau^{\prime}\right), q(\tau)\right) .
$$

Since for every $\left.x \in \operatorname{Im} q\right|_{\left[\tau^{\prime}, \tau\right]}$ we have that

$$
\frac{\|s(x)\|}{\omega(\xi(\tau))} \leq \frac{\|s(x)\|}{\omega\left(d_{F}\left(x, x_{0}\right)\right)} \leq C
$$

we obtain that

$$
\ell_{F}\left(\left.p\right|_{\left[\tau^{\prime}, \tau\right]}\right) \geq \frac{d_{F}\left(q\left(\tau^{\prime}\right), q(\tau)\right)}{C \cdot \omega(\xi(\tau))} .
$$

As a consequence we have that, for every $\tau^{\prime}<\tau$ in $[0, \delta]$ :

$$
\begin{aligned}
d_{F}\left(q(\tau), x_{0}\right) & \leq d_{F}\left(q\left(\tau^{\prime}\right), x_{0}\right)+C \cdot \omega(\xi(\tau)) \cdot \ell_{F}\left(\left.p\right|_{\left[\tau^{\prime}, \tau\right]}\right) \\
& \leq \xi\left(\tau^{\prime}\right)+C \cdot \omega(\xi(\tau)) \cdot \ell_{F}\left(\left.p\right|_{\left[\tau^{\prime}, \tau\right]}\right) .
\end{aligned}
$$

In order to establish (3.1), note that the inequality is clear if $\xi\left(\tau^{\prime}\right)=\xi(\tau)$. On the other hand, if $\xi\left(\tau^{\prime}\right)<\xi(\tau)$, there exists $\tau^{*} \in\left(\tau^{\prime}, \tau\right]$ such that $\xi(\tau)=$ $d_{F}\left(x_{0}, q\left(\tau^{*}\right)\right)$. In this case, by applying the above argument to $\left[\tau^{\prime}, \tau^{*}\right]$, we obtain that

$$
\begin{aligned}
\xi(\tau)=d_{F}\left(x_{0}, q\left(\tau^{*}\right)\right) & \leq \xi\left(\tau^{\prime}\right)+C \cdot \omega\left(\xi\left(\tau^{*}\right)\right) \cdot \ell_{F}\left(\left.p\right|_{\left[\tau^{\prime}, \tau^{*}\right]}\right) \\
& \leq \xi\left(\tau^{\prime}\right)+C \cdot \omega(\xi(\tau)) \cdot \ell_{F}\left(\left.p\right|_{\left[\tau^{\prime}, \tau\right]}\right) .
\end{aligned}
$$

Now, given a partition $0=t_{0}<t_{1}<\cdots<t_{n}=\xi(\delta)$ of $[0, \xi(\delta)]$, since $\xi$ is continuous and non-decreasing we can find $0=\tau_{0}<\tau_{1}<\cdots<\tau_{n}=\delta$ such that $t_{i}=\xi\left(\tau_{i}\right)$, for $i=0, \ldots, n$. Then, by inequality (3.1), we have:

$$
\sum_{i=0}^{n-1} \omega\left(t_{i+1}\right)\left(t_{i+1}-t_{i}\right) \leq C \cdot \sum_{i=0}^{n-1} \ell_{F}\left(\left.p\right|_{\left[\tau_{i}, \tau_{i+1}\right]}\right)=C \cdot \ell_{F}\left(\left.p\right|_{[0, \delta]}\right) \leq C \cdot \ell_{F}(p) \text {. }
$$

Therefore, for every $\delta \in[0, b)$ we obtain that

$$
\int_{0}^{\xi(\delta)} \frac{d t}{\omega(t)} \leq C \cdot \ell_{F}(p)<\infty
$$

Since $\omega$ is a weight, we conclude that there exists some $r>0$ such that $\xi(\delta) \leq r$, for every $\delta \in[0, b)$. Therefore, for all $x \in \operatorname{Im} q$ we have:

$$
\frac{\|s(x)\|}{\omega(r)} \leq \frac{\|s(x)\|}{\omega\left(d_{F}\left(x, x_{0}\right)\right)} \leq C .
$$

Using equality (2.3) we get that, for every $t \in[0, b)$,

$$
\|\dot{q}(t)\| \leq\|s(q(t))\| \cdot\|\dot{p}(t)\| \leq C \cdot \omega(r) \cdot\|\dot{p}(t)\| .
$$


Then for each $\delta \in[0, b)$ we have

$$
\begin{aligned}
\ell_{F}\left(\left.q\right|_{[0, \delta]}\right)=\int_{0}^{\delta}\|\dot{q}(t)\| d t & \leq C \cdot \omega(r) \cdot \ell_{F}\left(\left.p\right|_{[0, \delta]}\right) \\
& \leq C \cdot \omega(r) \cdot \ell_{F}(p)<\infty .
\end{aligned}
$$

In conclusion, $\ell_{F}(q)<\infty$.

$(3 \Rightarrow 4)$ Suppose that $\ell_{F}(q)<\infty$. Let $\left\{t_{n}\right\} \subset[0, b)$ be any sequence such that $t_{n} \rightarrow b$. Since $\int_{0}^{t_{n}}\|\dot{q}(t)\| d t$ converges to $\ell_{F}(q)$, we obtain that $\left\{q\left(t_{n}\right)\right\}$ is a Cauchy sequence for the Finsler metric in the connected component $M_{0}$. By completeness, $\left\{q\left(t_{n}\right)\right\}$ is then convergent.

$(4 \Rightarrow 5)$ It is clear.

$(5 \Rightarrow 1)$ It follows directly from the fact that $\|s(x)\|>0$ for all $x \in M$ and the mapping $x \mapsto\|s(x)\|$ it is continuous on $M$ (see $[\mathbf{1 0}, \S 2]$ ).

Let $f: M \rightarrow N$ be a split submersion of class $C^{2-}$ and let $s(\cdot)$ be a right inverse of $d f(\cdot)$ of class $C^{1-}$. We shall say that $f$ has the bounded path-lifting property with respect to $s(\cdot)$ if, for each $C^{1}$ path $p:[0,1] \rightarrow N$ and each $0<b \leq 1$, we have that every $C^{1}$ horizontal lifting $q:[0, b) \rightarrow M$ of $p$ satisfies the equivalent conditions of Lemma 3.1. With this terminology Lemma 3.1(4) gives that the bounded path-lifting property implies the continuation property for horizontal liftings. Thus combining with Theorem 2.11 we get at once the following:

Theorem 3.2. Let $M$ and $N$ be $C^{2}$ Finsler manifolds, $M$ complete and $N$ connected, and let $f: M \rightarrow N$ be a $C^{2-}$ split submersion. Suppose that $f$ has the bounded path-lifting property with respect to some $C^{1-}$ right inverse $s(\cdot)$ of $d f(\cdot)$. Then $f$ is a fiber bundle.

Now from Theorem 3.2 we can easily deduce the following corollary, related to a result by Earle and Eells [4, Theorem 3C]. There, Earle and Eells do not assume that $N$ is connected, but they assume instead that $f$ is surjective.

Corollary 3.3. Let $M$ and $N$ be $C^{2}$ Finsler manifolds, $M$ complete and $N$ connected. Let $f: M \rightarrow N$ be a $C^{2-}$ split submersion and let $s(\cdot)$ be a $C^{1-}$ right inverse of $d f(\cdot)$. Suppose that for every $y_{0} \in N$, there exist a neighborhood $V_{0}$ of $y_{0}$ in $N$ and $C_{0}>0$ such that, for every $x \in f^{-1}\left(V_{0}\right)$, $\|s(x)\| \leq C_{0}$. Then, $f$ has the bounded path-lifting property with respect to $s(\cdot)$, and therefore it is a fiber bundle.

Proof. Let $p:[0,1] \rightarrow N$ be a $C^{1}$ path. By compactness, we can find a finite open covering $\operatorname{Im} p \subset V_{1} \cup \ldots \cup V_{n}$ and some $C>0$ such that $\|s(x)\| \leq C$, for every $x \in f^{-1}\left(V_{i}\right)$, for all $i=1, \ldots, n$. If $q:[0, b) \rightarrow M$ is such that $f \circ q=p$ over $[0, b)$, we obtain that $\sup \{\|s(x)\|: x \in \operatorname{Im} q\} \leq C$. Thus every lifting of $p$ satisfies Condition (1) of Lemma 3.1. 
Next we give a result for connected manifolds, in terms of the Hadamard integral condition. Here we denote by $\overline{B_{t}\left(x_{0}\right)}$ the closed ball with center $x_{0}$ and radius $t$, with respect to the Finsler metric $d_{F}$ of $M$. We note that Corollary 3.4 below was obtained by Plastock [13, Theorem 3.2] for the special case of Fredholm submersions with positive index between Banach spaces.

Corollary 3.4. Let $M$ and $N$ be $C^{2}$ connected Finsler manifolds, $M$ complete. Suppose that $f$ is a $C^{2-}$ split submersion that satisfies

$$
\int_{0}^{\infty} \underset{x \in \overline{B_{t}\left(x_{0}\right)}}{\inf }\|s(x)\|^{-1} d t=\infty
$$

for some $C^{1-}$ right inverse $s(\cdot)$ of $d f(\cdot)$ and some $x_{0} \in M$. Then $f$ has the bounded path-lifting property with respect to $s(\cdot)$, and therefore it is a fiber bundle.

Proof. We can consider the weight $\omega(t):=\left[\inf \left\{\|s(x)\|^{-1}: x \in \overline{B_{t}\left(x_{0}\right)}\right\}\right]^{-1}$, defined for $t \geq 0$. It is not difficult to check that $\|s(x)\| \cdot \omega\left(d\left(x, x_{0}\right)\right)^{-1} \leq 1$, for each $x \in M$. In particular for every path $p:[0,1] \rightarrow N$, every lifting satisfies Condition (2) of Lemma 3.1.

Remark 3.5. In the special case that $M$ and $N$ are Riemannian manifolds (hence modelled on Hilbert spaces) we can use the right inverse of $d f(\cdot)$ considered in Remark 2.2, that is, $s_{0}(x)=\left(\left.d f(x)\right|_{[\operatorname{ker} d f(x)]^{\perp}}\right)^{-1}=$ $d f(x)^{*}\left[d f(x) d f(x)^{*}\right]^{-1}$. In this case the norm $\left\|s_{0}(x)\right\|$ can be computed explicitly, by means of the following formula (see Remark 4.1 below):

$$
\begin{aligned}
\left\|s_{0}(x)\right\|^{-1} & =\inf \left\{\|d f(x)(u)\|: u \in[\operatorname{ker} d f(x)]^{\perp} ;\|u\|=1\right\} \\
& =\inf \left\{\left\|d f(x)^{*}(v)\right\|: v \in T_{f(x)} N ;\|v\|=1\right\} .
\end{aligned}
$$

To finish this section, we specialize to the case where $f: M \rightarrow N$ is a local diffeomorphism. Of course, in this case the only choice for a right inverse of $d f(\cdot)$ is $s(x)=d f(x)^{-1}$, and every lifting is horizontal. Thus we obtain:

Theorem 3.6. Let $M$ and $N$ be $C^{2}$ Finsler manifolds, $M$ complete and $N$ connected. Let $f: M \rightarrow N$ be a map of class $C^{2-}$ and suppose that, for every $x \in M, d f(x) \in \operatorname{Isom}\left(T_{x} M ; T_{f(x)} N\right)$. The following statements are equivalent:

(1) $f$ is a covering projection.

(2) $f$ has the continuation property.

(3) $f$ has the bounded path-lifting property with respect to $s(x)=d f(x)^{-1}$. If in addition, we assume that either $N$ is simply connected or $\pi_{1}(M)=$ $\pi_{1}(N)$ is finite, then the previous conditions are also equivalent to the following:

(4) For every compact subset $K \subset N$, there is a constant $C>0$ such that $\left\|d f(x)^{-1}\right\| \leq C$, for every $x \in f^{-1}(K)$. 
(5) $f$ is a proper map.

(6) $f$ is a closed map.

(7) $f$ is a homeomorphism.

Proof. The equivalences (1)-(3) can be deduced by combining Corollary 2.4 and Lemma 3.1. For the equivalences (4)-(7), consider Corollary 2.9, and use for example [16, Corollary 2.4.7].

\section{Submersions with uniformly split kernels}

A map $f: M \rightarrow N$ of class $C^{1}$ between $C^{1}$ Finsler manifolds is said to have uniformly split kernels if there is a constant $k>0$ such that for each $x \in M$, there exists a projection $P_{x} \in \mathcal{L}\left(T_{x} M, T_{x} M\right)$ with $\operatorname{ker} P_{x}=\operatorname{ker} d f(x)$ and $\left\|P_{x}\right\|_{x} \leq k$, where $\|\cdot\|_{x}$ denotes the norm induced by the Finsler structure $\|\cdot\|$ on $T M$. This concept was introduced by Rabier in [14]. As shown in [14, Proposition 3.1], the map $f$ has uniformly split kernels, for example, in the following cases:

(i) When $M$ is a Riemannian manifold (hence modelled on a Hilbert space);

(ii) when $N$ is finite-dimensional;

(iii) when $f$ is a Fredholm submersion of nonnegative index.

On the other hand, for a linear operator $T \in \mathcal{L}(E, F)$ between Banach spaces, Rabier [14] sets the quantity

$$
\nu(T)=\inf _{\left\|y^{*}\right\|=1}\left\|T^{*} y^{*}\right\|,
$$

where $T^{*} \in \mathcal{L}\left(F^{*}, E^{*}\right)$ denotes the transpose of $T$. It is easy to see that if $T \in \operatorname{Isom}(E, F)$, then $\nu(T)=\left\|T^{-1}\right\|^{-1}$. More generally, we have the following:

Remark 4.1. Let $T: E \rightarrow F$ be a linear onto map between Banach spaces, and consider the canonical isomorphism $\widehat{T}: E / \operatorname{ker} T \rightarrow F$. Then $\nu(T)=$ $\left\|\widehat{T}^{-1}\right\|^{-1}$. Indeed, if $\pi: E \rightarrow E / \operatorname{ker} T$ denotes the quotient map, we have that $T=\widehat{T} \circ \pi$ and $T^{*}=\pi^{*} \circ \widehat{T}^{*}$. Now consider $(\operatorname{ker} T)^{0}=\left\{x^{*} \in E^{*}\right.$ : $\left.x^{*}(\operatorname{ker} T)=0\right\}$. We know that $\pi^{*}:(E / \operatorname{ker} T)^{*} \rightarrow(\operatorname{ker} T)^{0}$ is an isometry and $T^{*}: F^{*} \rightarrow(\operatorname{ker} T)^{0}$ is an isomorphism. Therefore,

$$
\nu(T)=\inf _{\left\|y^{*}\right\|=1}\left\|T^{*} y^{*}\right\|=\inf _{\left\|y^{*}\right\|=1}\left\|\widehat{T}^{*} y^{*}\right\|=\left\|\left(\widehat{T}^{*}\right)^{-1}\right\|^{-1}=\left\|\widehat{T}^{-1}\right\|^{-1} .
$$

Next we are going to see that, when $f: M \rightarrow N$ is a $C^{2-}$ submersion with uniformly split kernels, it is possible to construct a $C^{1-}$ right inverse $s(\cdot)$ of $d f(\cdot)$ satisfying a special boundedness condition. We know that, for every $x \in$ $M$, there exists a projection $P_{x} \in \mathcal{L}\left(T_{x} M, T_{x} M\right)$ with ker $P_{x}=\operatorname{ker} d f(x)$ and $\left\|P_{x}\right\|_{x} \leq k$, for some uniform constant $k>0$. For each $x \in M$, consider the quotient map $\pi_{x}: T_{x} M \rightarrow T_{x} M / \operatorname{ker} d f(x)$ and the canonical isomorphism 
$\widehat{d f(x)}: T_{x} M / \operatorname{ker} d f(x) \rightarrow T_{f(x)} N$. If we define $\sigma_{x}(u+\operatorname{ker} d f(x))=P_{x}(u)$, we obtain that $\sigma_{x}: T_{x} M / \operatorname{ker} d f(x) \rightarrow T_{x} M$ is a right inverse of $\pi_{x}$ with $\left\|\sigma_{x}\right\| \leq k$, and

$$
s_{x}:=\sigma_{x} \circ \widehat{d f(x)}^{-1}: T_{f(x)} N \rightarrow T_{x} M
$$

is a right inverse of $d f(x)$ with $\left\|s_{x}\right\| \leq k \cdot\left\|\widehat{d f(x)}^{-1}\right\|=k \cdot \nu(d f(x))^{-1}$. Now using a partition of unity of class $C^{1-}$, it is possible to obtain the following result, due to Rabier (it is essentially [14, Proposition 3.1] with minor modifications):

Lemma 4.2. Let $M$ and $N$ be $C^{2}$ Finsler manifolds and let $f: M \rightarrow N$ be a $C^{2-}$ submersion with uniformly split kernels. Then there exist a $C^{1-}$ right inverse $s(\cdot)$ of $d f(\cdot)$ and a constant $k>0$ such that, for every $x \in M$ :

$$
\|s(x)\| \leq k \cdot \nu(d f(x))^{-1} .
$$

Now we introduce a path-lifting property whose definition does not involve explicitly a $C^{1-}$ right inverse. We shall say that $f: M \rightarrow N$ has the $\nu$-bounded path-lifting property if for every $C^{1}$ path $p:[0,1] \rightarrow N$, every $b \in(0,1]$ and every $C^{1}$ path $q:[0, b) \rightarrow M$ with $f \circ q=p$ over $[0, b)$, there exist a weight $\omega$, some $C>0$ and some $x_{0}$ in the connected component of $M$ containing $\operatorname{Im} q$, such that:

$$
\sup \left\{\frac{\nu(d f(x))^{-1}}{\omega\left(d_{F}\left(x, x_{0}\right)\right)}: x \in \operatorname{Im} q\right\} \leq C .
$$

By Lemma 4.2, if $f: M \rightarrow N$ is a $C^{2-}$ submersion with uniformly split kernels satisfying the $\nu$-bounded path-lifting property, then $f$ has the bounded path-lifting property for some $C^{1-}$ right inverse of $d f(\cdot)$. Thus from Theorem 3.2 it follows at once:

Theorem 4.3. Let $M$ and $N$ be $C^{2}$ Finsler manifolds, $M$ complete and $N$ connected. Let $f: M \rightarrow N$ be a $C^{2-}$ submersion with uniformly splits kernels. If $f$ has the $\nu$-bounded path-lifting property, then $f$ is a fiber bundle.

A remarkable class of submersions satisfying the $\nu$-bounded path-lifting property are the strong submersions in the sense of Rabier. Recall from [14] that a $C^{1}$ submersion $f: M \rightarrow N$ is said to be strong submersion if there is no sequence $\left\{x_{n}\right\}$ in $M$ such that $\lim _{n \rightarrow \infty} f\left(x_{n}\right)$ exists in $N$ and $\lim _{n \rightarrow \infty} \nu\left(d f\left(x_{n}\right)\right)=0$. Equivalently, $f$ is a strong submersion if, and only if, for every compact subset $K$ of $N$ there exists $\alpha_{K}>0$ such that $\inf \{\nu(d f(x))$ : $\left.x \in f^{-1}(K)\right\} \geq \alpha_{K}$. It is then clear that every strong submersion satisfies the $\nu$-bounded path-lifting property, and therefore the result that every $C^{2-}$ strong submersion with uniformly split kernels is a fiber bundle [14, Theorem 4.1] can be seen like a consequence of Theorem 4.3. (In fact, Rabier also considers an open set $U \subset M$, and works with $f: U \rightarrow N$, but introduces 
and extra assumption over $f$; the same can be done here to obtain the desired result.)

Next we give a result for submersions with uniformly split kernels between connected manifolds, using a Hadamard integral condition in terms of $\nu(d f(\cdot))$. The proof is similar to that of Corollary 3.4.

Corollary 4.4. Let $M$ and $N$ be $C^{2}$ connected Finsler manifolds, $M$ complete. Suppose that $f$ is a $C^{2-}$ submersion with uniformly split kernels, such that

$$
\int_{0}^{\infty} \inf _{x \in B_{t}\left(x_{0}\right)} \nu(d f(x)) d t=\infty,
$$

for some $x_{0} \in M$. Then $f$ is a fiber bundle.

In what follows, we are going to show that the different conditions that we have considered along this section are, in fact, nonequivalent. This can be seen by means of quite simple examples.

Example 4.5. Consider a $C^{\infty}$-diffeomorphism $\psi: \mathbb{R} \rightarrow \mathbb{R}$ such that $\inf \left\{\left|\psi^{\prime}(t)\right|: t \in \mathbb{R}\right\}=0$, and define the $C^{\infty}$ submersion $f: \mathbb{R} \rightarrow \mathbb{S}^{1}$ by $f(t)=(\cos \psi(t), \sin \psi(t))$. In this case $\nu(d f(t))=\left|\psi^{\prime}(t)\right|$ for every $t \in \mathbb{R}$, and therefore $f$ is not a strong submersion. On the other hand, since $f$ is a covering, $f$ has the $\nu$-bounded path-lifting property.

In the next two examples, we consider a $C^{\infty}$-diffeomorphism $\phi:(0, \infty) \rightarrow$ $(0, \infty)$, and we define $f: \mathbb{R} \times(0, \infty) \rightarrow \mathbb{R}$ by $f(x, y)=x \cdot \phi(y)$. Then $f$ is a $C^{\infty}$ submersion, and it is not difficult to check that $\nu(d f(x, y))=$ $\left[\phi(y)^{2}+\phi^{\prime}(y)^{2} x^{2}\right]^{\frac{1}{2}}$, for every $(x, y) \in \mathbb{R} \times(0, \infty)$.

Example 4.6. Consider here $\phi(y)=y^{-1}$ and define $f: \mathbb{R} \times(0, \infty) \rightarrow \mathbb{R}$ by $f(x, y)=x y^{-1}$. Since in this case $\nu(d f(x, y))=\left[y^{-2}+y^{-4} x^{2}\right]^{\frac{1}{2}}$, we have that $f$ is not a strong submersion. On the other hand, we are going to see that $f$ satisfies the Hadamard integral condition of Corollary 4.4. Indeed, for $z_{0}=(0,1) \in \mathbb{R} \times(0, \infty)$ we have that $d_{F}\left((x, y), z_{0}\right)=\left[x^{2}+(y-1)^{2}\right]^{\frac{1}{2}}$. Note that if $d_{F}\left((x, y), z_{0}\right) \leq r$, then $y \leq 1+r$. Therefore for every $(x, y) \in \overline{B_{r}\left(z_{0}\right)}$ we have that $\nu(d f(x, y)) \geq \frac{1}{y} \geq \frac{1}{1+r}$, and as a consequence:

$$
\int_{0}^{\infty} \inf _{(x, y) \in \overline{B_{r}\left(z_{0}\right)}} \nu(d f(x, y)) d r \geq \int_{0}^{\infty} \frac{d r}{1+r}=\infty .
$$

In our final example we exhibit a submersion $f$ which does not satisfy the $\nu$-bounded path-lifting property (and in particular $f$ is not a strong submersion), and nevertheless $f$ satisfies the bounded path-lifting property for some right inverse $s(\cdot)$ of $d f(\cdot)$.

Example 4.7. Consider here $\phi(y)=y^{-2}$ and define $f: \mathbb{R} \times(0, \infty) \rightarrow \mathbb{R}$ by $f(x, y)=x y^{-2}$. In this case $\nu(d f(x, y))=\left[y^{-4}+4 y^{-6} x^{2}\right]^{\frac{1}{2}}$, and for $z_{0}=$ 
$(0,1) \in \mathbb{R} \times(0, \infty)$ we have that $d_{F}\left((x, y), z_{0}\right)=\left[x^{2}+(y-1)^{2}\right]^{\frac{1}{2}}$. Consider the paths $p:[0,1] \rightarrow \mathbb{R}$ given by $p(t)=t$ and $q:(0,1] \rightarrow \mathbb{R} \times(0,+\infty)$ given by $q(t)=\left(1, t^{-\frac{1}{2}}\right)$. It is clear that $f(q(t))=p(t)$, for every $t \in(0,1]$. Furthermore, it is easy to check that $\nu(d f(q(t))) \leq \sqrt{5} t$, and $d_{F}\left(q(t), z_{0}\right) \leq$ $\sqrt{2} t^{-\frac{1}{2}}$, for every $t \in(0,1]$. Suppose now that $f$ satisfies the $\nu$-bounded path-lifting property. Then there exist a constant $C>0$ and a weight $\omega$ such that $C \cdot \nu(d f(q(t))) \geq \omega\left(d_{F}\left(q(t), z_{0}\right)\right)^{-1}$, for every $t \in(0,1]$. Taking $r=\sqrt{2} t^{-2}$ we obtain that $\omega(r)^{-1} \leq 2 \sqrt{5} C r^{-2}$, for every $r \in[\sqrt{2}, \infty)$. As a consequence,

$$
\int_{\sqrt{2}}^{\infty} \frac{d r}{\omega(r)} \leq \int_{\sqrt{2}}^{\infty} \frac{C}{r^{2}} d r<\infty
$$

which is a contradiction with the fact that $\omega$ is a weight. On the other hand, consider the right inverse $s(\cdot)$ of $d f(\cdot)$ defined, for every $(x, y) \in \mathbb{R} \times(0, \infty)$ by $s(x, y): \mathbb{R} \rightarrow \mathbb{R} \times \mathbb{R}$, where $s(x, y)(r)=\left(r y^{2}, 0\right)$. Let $p:[0,1] \rightarrow \mathbb{R}$ be a $C^{1}$ path, let $0<b \leq 1$, and consider an horizontal lifting $q:[0, b) \rightarrow \mathbb{R} \times(0, \infty)$. If we denote $q(t)=\left(q_{1}(t), q_{2}(t)\right)$, it is easy to see that $\dot{q}_{1}(t)=q_{2}(t)^{2} \dot{p}(t)$ and $\dot{q}_{2}(t)=0$, for every $t \in[0, b)$. Therefore $q_{2}$ is constant, say $q_{2}(t)=c$, and $q_{1}(t)=c^{2} p(t)$, for every $t \in[0, b)$. Then it is clear that $q$ can be continuously extended to $[0, b]$. In this way we see that $f$ has the bounded path-lifting property with respect to $s(\cdot)$.

Acknowledgements. It is a great pleasure to thank Professors Javier Gómez Gil and María Isabel Garrido for some valuable conversations concerning this paper.

\section{References}

[1] R. Abraham, J.E. Marsden and T. Ratiu, Manifolds, Tensor Analysis and Applications, Applied Mathematical Sciences, 75, Springer-Verlag, 1988, MR 0960687 (89f:58001), Zbl 0875.58002.

[2] M.S. Berger and R.A. Plastock, On the singularities of nonlinear Fredholm operators of positive index, Proc. Amer. Math. Soc., 79(2) (1980), 373-380, MR 0565342 (81d:58011), Zbl 0444.47044.

[3] F.E. Browder, Covering spaces, fiber spaces and local homeomorphism, Duke Math. J., 21 (1954), 329-336, MR 0062431 (15,978a), Zbl 0056.16602.

[4] C.J. Earle and J. Eells, Foliations and fibrations, J. Differential Geom., 1 (1967), 61-69, MR 0215320 (35 \#6161), Zbl 0162.54101.

[5] C. Ehresmann, Les connexions infinitésimales dans un espace fibré différentiable, Colloque de Topologie, Bruxelles, 1950, Georges Thone, Liège; Masson et Cie., Paris, 1951, 29-55, MR 0042768 (13,159e), Zbl 0054.07201.

[6] R. Engelking, General Topolgy, PWN Polish Scientific Publishers, Warszawa, 1977.

[7] O. Gutú and J.A. Jaramillo, Global homeomorphisms and covering projections on metric spaces. Preprint. 
[8] J. Hadamard, Sur les tranformations ponctuelles, Bull. Soc. Math. France, 34 (1906), 71-84.

[9] M.W. Hirsch, Differential Topology, Graduate Texts in Mathematics, 33, SpringerVerlag, 1976, MR 0448362 (56 \#6669), Zbl 0356.57001.

[10] R.S. Palais, Lusternik-Schnirelman theory on Banach manifolds, Topology, 5 (1966), 115-132, MR 0259955 (41 \#4584), Zbl 0143.35203.

[11] T. Parthasarathy, On Global Univalence Theorems, Lecture Notes in Mathematics, 977, Springer-Verlag, 1983, MR 0694845 (84m:26019), Zbl 0506.90001.

[12] R.A. Plastock, Homeomorphisms between Banach spaces, Trans. Amer. Math. Soc., 200 (1974), 169-183, MR 0356122 (50 \#8593), Zbl 0291.54009.

[13] Nonlinear mappings that are globally equivalent to a projection, Trans. Amer. Math. Soc., 275(1) (1983), 373-380, MR 0678357 (84b:58017), Zbl 0525.47047.

[14] P.J. Rabier, Ehresmann fibrations and Palais-Smale conditions for morphisms of Finsler Manifolds, Ann. of Math., 146 (1997), 647-691, MR 1491449 (98m:58020), Zbl 0919.58003.

[15] W.C. Rheinboldt, Local mapping relations and global implicit functions theorems, Trans. Amer. Math. Soc., 138 (1969), 183-198, MR 0240644 (39 \#1990), Zbl 0175.45201.

[16] E.H. Spanier, Algebraic Topology, McGraw Hill, 1966, MR 0210112 (35 \#1007), Zbl 0145.43303.

Received July 9, 2003 and revised October 24, 2003. The first author was supported by CONACyT (México) Grant 119933. The second author was supported in part by DGES (Spain) BFM2000-0609.

Departamento de Análisis Matemático

Universidad Complutense de Madrid

28040 MADRID

SPAIN

Centro de Investigación en Matemáticas

Universidad Autónoma del Estado de Hidalgo

42074 PaChuca Hidalgo

MÉXICO

E-mail address: olivia@uaeh.reduaeh.mx

Departamento de Análisis Matemático

Universidad Complutense De Madrid

28040 MADRID

SPAIN

E-mail address: jaramil@mat.ucm.es 